Research article

Open Access

\title{
The Reaction of Cyanoacetic Acid Hydrazide with 2-Acetylfuran: Synthesis of Coumarin, Pyridine, Thiophene and Thiazole Derivatives with Potential Antimicrobial Activities
}

\author{
Rafat M. Mohareb * 1,2, Elham Ezz El-ARAB ${ }^{3}$, Karam A. El-SharkaWy ${ }^{1}$ \\ ${ }^{1}$ Faculty of pharmacy, October University for Modern Sciences \& Arts, Elwahaat Road, October City, A. R. \\ Egypt. \\ ${ }^{2}$ Faulty of Science, Chemistry Department, Cairo University, A. R. Egypt \\ ${ }^{3}$ National Organization of Drug Control \& Research (NODCAR), P.O. 29, Cairo, A. R. Egypt \\ * Corresponding author. E-mail: raafat_mohareb@yahoo.com (R. M. Mohareb)
}

Sci Pharm. 2009; 77: 355-366

doi:10.3797/scipharm.0901-20

Published: $\quad$ March $14^{\text {th }} 2009$

Accepted: $\quad$ March $11^{\text {th }} 2009$

Received: January $18^{\text {th }} 2009$

This article is available from: http://dx.doi.org/10.3797/scipharm.0901-20

(C) Mohareb et al.; licensee Österreichische Apotheker-Verlagsgesellschaft m. b. H., Vienna, Austria.

This is an Open Access article distributed under the terms of the Creative Commons Attribution License (http://creativecommons.org/licenses/by/3.0/), which permits unrestricted use, distribution, and reproduction in any medium, provided the original work is properly cited.

\begin{abstract}
The hydrazide-hydrazone derivative 1 was formed through the reaction of cyanoacetic acid hydrazide with 2-acetylfuran. Compound $\mathbf{1}$ underwent a series of hetrocyclization reactions through its reaction with different chemical reagents to produce arylidene, coumarin, aryl hydrazone, pyridine, thiophene and thiazole derivatives 2-10. The MIC values for the newly synthesized products were tested against E. coli, B. cereus, B. subtilis and C. albicans compared with ampicilline and cycloheximide as reference drugs.
\end{abstract}

\section{Keywords}

Pyridine • Thiophene • Thiazole • Antimicrobial

\section{Introduction}

It is well known that the hydrazone group plays an important role for the antimicrobial activity. Furthermore, a number of hydrazide-hydrazone claimed to possess interesting antibacterial-antifungal [1-3] anticonvulsant [4,5] anti-inflammatory [6, 7] antimalarial [8] and antituberculosis activities [9-15]. We report here the synthesis of a series of 
hydrazide-hydrzones via the reaction of cyanoacetylhydrazine with 2-acetylfuran with the aim of obtaining new hydrazide-hydrazones with wide spectrum of pharamaceutical applications,.

\section{Results and Discussions}

Cyanoacetylhydrazine reacts with 2-acetylfuran to give the hydrazide-hydrazone derivative 1. The structure of compound 1 was established on the basis of analytical and spectral data. Thus ${ }^{1} \mathrm{H}$ NMR spectrum showed the presence of a singlet at $\delta 1.01 \mathrm{ppm}$ corresponding to $\mathrm{CH}_{3}$ group, a singlet at $\delta 3.31 \mathrm{ppm}$ for $\mathrm{CH}_{2}$ group, a multiplet at $\delta$ 6.42$7.29 \mathrm{ppm}$ for the furan protons and a singlet at $\delta 7.90 \mathrm{ppm}$ for an $\mathrm{NH}$ group. Moreover, the ${ }^{13} \mathrm{C}$ NMR data showed the presence of $\delta 18.7\left(\mathrm{CH}_{3}\right), 27.9\left(\mathrm{CH}_{2}\right), 110.3,129.6,137.2$, 143.9 (furan $\mathrm{C}), 156.9(\mathrm{C}=\mathrm{N}), 168.4(\mathrm{C}=\mathrm{O})$. Further elucidation for the structure of 1 was obtained through studying its chemical reactivity through some chemical reagents. Thus, the reaction of 1 with aromatic aldehydes either benzaldehyde, 4-chlorobenzaldehyde or 4-methoxybenzaldehyde gave the benzalidene derivatives $2 a-c$, respectively. Analytical and spectral data are in agreement with the proposed structures. On the other hand, the reaction of 1 with salicylaldehyde gave the coumarin derivative 3 . The reaction goes in analogy with the reported literature $[16,17]$. The reaction of 1 with either benzenediazonium chloride, 4-chlorobenzenediazonium chloride and 4-methylbenzenediazonium chloride gave the aryl hydrazone derivatives $4 \mathbf{a}-\mathbf{c}$, respectively.

The reaction of compound 1 with cinnamonitrile derivatives was studied. Thus, the reaction of 1 with either a-cyanocinnamonitrile or ethyl a-cyanocinnamate gave the pyridine derivatives $\mathbf{6 a}$ and $\mathbf{6 b}$, respectively. The reaction took place through the intermediate formation of $\mathbf{5 a , b}$. Structures of $\mathbf{6 a}$ and $\mathbf{6 b}$ were based on analytical and spectral. Thus, the ${ }^{1} \mathrm{H}$ NMR spectrum of $6 \mathrm{a}$ showed the presence of a singlet at $\delta 1.05$ corresponding to the $\mathrm{CH}_{3}$ group, a singlet at $\delta 4.87$ for an $\mathrm{NH}_{2}$ group, a multiplet at $\delta 7.28-7.41$ phenyl and furan protons. Further confirmation for the structures of $\mathbf{6 a}$ and $\mathbf{6 b}$ were obtained through their synthesis via another reaction root. Thus, the reaction of compound $2 \mathbf{a}$ with either malononitrile or ethyl cyanoacetate gave the same pyridine derivatives $\mathbf{6 a}$ and $\mathbf{6 b}$, respectively (m.p., mixed m.p. and fingerprint IR). The reaction of $\mathbf{1}$ with either malononitrile or ethyl cyanoacetate gave the pyridin-2-one derivatives $\mathbf{7 a}$ and $\mathbf{7 b}$, respectively. Analytical and spectral data are consistent with the proposed structures.

The reaction of 1 with either malononitrile or ethyl cyanoacetate and elemental sulfur in the presence of triethyl amine gave the thiophene derivatives $\mathbf{8 a}$ and $\mathbf{8 b}$, respectively. The reaction goes in parallel to the reported Gewald's thiophene synthesis [18]. Similarly, the reaction of 1 with cyclohexanone and elemental sulfur gave the 4,5,6,7-tetrahydrobenzothiophene derivative $\mathbf{9}$. Formation of $\mathbf{9}$ took place according the similar reported reactions of cyclohexanone with methylene reagents and elemental sulfur [19]. On the other hand the reaction of $\mathbf{1}$ with elemental sulfur and phenylisothiocyanate gave the thiazole derivative 10. Formation of the latter product took place in parallel to the reported Hanzesch reported reaction [20]. Structures of compounds 9 and 10 were based on analytical and spectral data. 
$\overbrace{\mathrm{CN}}^{\mathrm{O}} \mathrm{NHNH}_{2}+$<smiles>CC(=O)c1ccco1</smiles><smiles>CC</smiles>

1

$\mathrm{Ar}-\mathrm{CHO}$

$$
\mathrm{Ar}=\mathrm{Ph}
$$

$\mathrm{Ar}=4-\mathrm{Cl}-\mathrm{Ph}$

$\mathrm{Ar}=4-\mathrm{MeO}-\mathrm{Ph}$

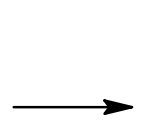

1<smiles>O=Cc1ccccc1O</smiles>

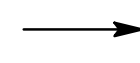

1<smiles>C/C(=N\NC(=O)/C(C#N)=C/[In])c1ccco1</smiles>

2a: $\mathrm{Ar}=\mathrm{Ph}$

2b: $\mathrm{Ar}=4-\mathrm{Cl}-\mathrm{Ph}$

2c: $\mathrm{Ar}=4-\mathrm{MeO}-\mathrm{Ph}$<smiles>C/C(=N\NC(=O)CC#N)c1ccco1</smiles>

1<smiles>C/C(=N\NC(=O)c1cc2ccccc2oc1=O)c1ccco1</smiles>
4a: $\mathrm{Ar}=\mathrm{Ph}$
4b: $\mathrm{Ar}=4-\mathrm{Cl}-\mathrm{Ph}$
4c: $\mathrm{Ar}=4-\mathrm{MeO}-\mathrm{Ph}$

3<smiles>C/C(=N\NC(=O)/C(C#N)=N/N[Al])c1ccco1</smiles>

Sch. 1.

$$
\mathrm{Ar}=4-\mathrm{Cl}-\mathrm{Ph}
$$$$
\mathrm{Ar}=4-\mathrm{MeO}-\mathrm{Ph}
$$

According to the results shown in Table 1, only two compounds $\mathbf{1}$ and $\mathbf{4 b}$ are moderately active against $E$. coli in the concentrations tested (average MIC $12.5 \mu \mathrm{gmL}^{-1}$ ), the cyanoacetyl and 4-chlorophenyl derivatives of furan moieties being responsible for such activity. However, compound $\mathbf{1}$ was the higher active compound. Compounds $\mathbf{2 a}, \mathbf{6} \mathbf{b}$ and $\mathbf{7 b}$ are highly active compounds against $B$. Cereus. Also compound $\mathbf{6 b}$ showed the highest activity towards $B$. subtilis with respect to ampicilline. The compounds $\mathbf{2 c}, \mathbf{4 a}, \mathbf{6 a}$ and $\mathbf{8 b}$ shows the highest activity against $C$. albicans. Comparing the SAR of compounds $6 \mathbf{a}, \mathbf{b}$ compound $\mathbf{6 b}$ with the Ethylester group showed the highest activity towards $B$. subtilis. On the other hand considering the hydrazone derivatives $\mathbf{4 a - c}$, the phenylhydrazone 
compound $4 \mathrm{a}$ showed the highest activity towards C. albicans. Substitution with 4-chloro group as in $\mathbf{4 b}$ showed no activity towards $C$. albicans. Compounds $\mathbf{6 a , b}$ and $7 \mathbf{a}, \mathbf{b}$ (the substituted hydrazone derivatives) one can notice that the activity against $B$. cereus was increased due to the existence of the pyridine ring, but compounds $\mathbf{6 b}$ and $\mathbf{7 b}$ are higher active than the corresponding $\mathbf{6 a}$ and $\mathbf{7 a}$ due to the presence of the ethyl pyridine-3carboxylate moiety and hydroxy group respectively. Finally compound $\mathbf{6} \mathbf{b}$ is the only highest active against $B$. subtilis due to the presence of the ethyl pyridine-3-carboxylate moiety.

1

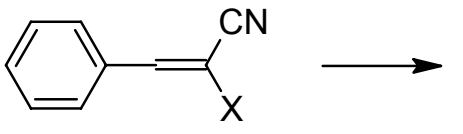

$\mathrm{X}=\mathrm{CN}$

$\mathrm{X}=$ COOEt

$2 a$<smiles>[Y]C[CH]</smiles>

$\mathrm{X}=\mathrm{CN}$

$X=$ COOEt

1<smiles>[X]CC[NH]</smiles>

$\mathrm{X}=\mathrm{CN}$

$X=$ COOEt

1<smiles>[X]CC#N</smiles>

$\mathrm{X}=\mathrm{CN}$

$X=$ COOEt

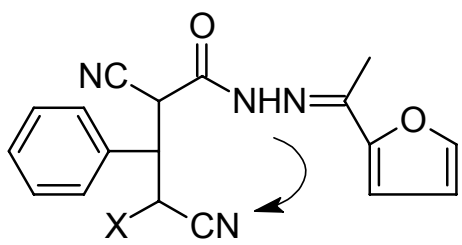

$5 a, b$<smiles>CC1([V])CCCCC1</smiles><smiles>[X]c1c(-c2ccccc2)c(C#N)c(=O)n(/N=C(/C)c2ccco2)c1N</smiles>

6a: $X=C N$

6b: $X=$ COOEt<smiles>[X]c1cc(N)c(C#N)c(=O)n1/N=C(/C)c1ccco1</smiles>

7a: $\mathrm{X}=\mathrm{NH}_{2}$

$7 \mathbf{b}: \mathrm{X}=\mathrm{OH}$<smiles>[X]c1c(N)sc(C(=O)NN=C(C)c2ccco2)c1N</smiles>

8a: $X=C N$

8b: $X=$ COOEt

Sch. 2. 


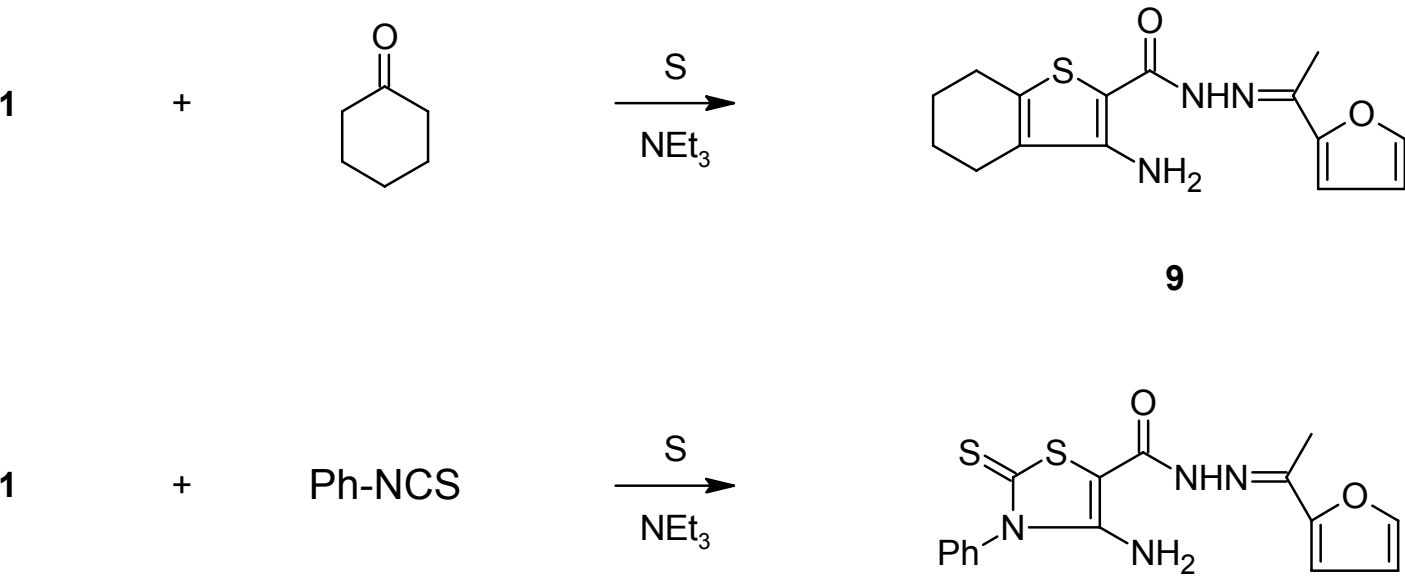

10

Sch. 3.

\section{Experimental}

\section{Synthetic methods, analytical and spectral data}

Melting points were determined on an electrothermal apparatus (Buchi 535, Switzerland) in an open capillary tube and are uncorrected. Elemental analyses were determined on a Yanaco CHN Corder elemental analyzer (Japan). IR spectra expressed in $\left(\mathrm{V} \mathrm{cm}^{-1}\right)$ were recorded in $\mathrm{KBr}$ pellets on a PA-9721 IR spectrophotometer (Shimadzu, Japan). ${ }^{1} \mathrm{H}$ NMR $\&{ }^{13} \mathrm{C}$ NMR spectra were obtained on a Jeol $300 \mathrm{MHz}$ (Japan) spectrometer in DMSO- $d_{6}$ as solvent, using TMS as internal reference and chemical shifts $(\delta)$ are expressed in ppm. Mass spectra were recorded on Kratos (75e-v) Ms Equipment (Germany). Synthetic pathways are presented in Schemes 1,2 and 3. Physicochemical and spectral data for the synthesized compounds are given. The antimicrobial activities of the tested compounds are given in the table. All compounds produced in this work are novel.

\section{2-Cyano-N '-(1-furan-2-ylethylidene)acetohydrazide (1)}

To a solution of 2-cyanoacetohydrazide $(1.0 \mathrm{~g}, 0.01 \mathrm{~mol})$ in 1,4-dioxane $(30 \mathrm{~mL})$ 2-acetylfuran $(1.7 \mathrm{~g}, 0.01 \mathrm{~mol})$ was added. The reaction mixture was heated under reflux for $1 \mathrm{~h}$ then left to cool. The solid product formed upon pouring onto ice/water was collected by filtration.

1. Colourless crystals of $\mathrm{mp} 155^{\circ} \mathrm{C}$ (ethanol). $\mathrm{MS}(\mathrm{m} / \mathrm{z}, \%)$ : $191\left(\mathrm{M}^{+}\right)$. IR $(\mathrm{KBr}), \mathrm{vcm}^{-1}$ : 3466, $3326(\mathrm{NH}), 3020$ (furan $\mathrm{CH}$ ), 2970, $2893\left(\mathrm{CH}_{3}, \mathrm{CH}_{2}\right), 2222(\mathrm{CN}), 1693(\mathrm{CO}), 1640$ $(\mathrm{C}=\mathrm{N}), 1632(\mathrm{C}=\mathrm{C}) .{ }^{1} \mathrm{H}$ NMR $\left(300 \mathrm{MHz}, \mathrm{DMSO}-\mathrm{d}_{6}, \mathrm{TMS}\right): \delta 1.01\left(\mathrm{~s}, 3 \mathrm{H}, \mathrm{CH}_{3}\right), 3.31(\mathrm{~s}, 2 \mathrm{H}$, $\left.\mathrm{CH}_{2}\right), 6.42-7.29(\mathrm{~m}, 3 \mathrm{H}$, furan $\mathrm{CH}), 7.90(\mathrm{~s}, 1 \mathrm{H}, \mathrm{NH}) .{ }^{13} \mathrm{C}$ NMR (300 MHz, DMSO- $\left.d_{6}, \mathrm{TMS}\right)$ : $\delta 18.7\left(\mathrm{CH}_{3}\right), 27.9\left(\mathrm{CH}_{2}\right), 110.3,129.6,137.2,143.9$ (furan $\left.\mathrm{C}\right), 156.9(\mathrm{C}=\mathrm{N}), 168.4(\mathrm{C}=\mathrm{O})$. Anal. Calcd. for $\mathrm{C}_{9} \mathrm{H}_{9} \mathrm{~N}_{3} \mathrm{O}_{2}: \mathrm{C}, 56.54 ; \mathrm{H}, 4.74 ; \mathrm{N}, 21.98$. Found: $\mathrm{C}, 56.44 ; \mathrm{H}, 4.61 ; \mathrm{N}, 22.05$.

\section{2-Cyano-N '-(1-furan-2-ylethylidene)-3-phenylprop-2-enehydrazide (2a),} 3-(4-chlorophenyl)-2-cyano-N '-(1-furan-2-ylethylidene)prop-2-enehydrazide (2b) and 


\section{2-cyano-N'-(1-furan-2-ylethylidene)-3-(4-methoxyphenyl)prop-2-enehydrazide (2c) N'-(1-furan-2-ylethylidene)-2-oxo-2H-chromene-3-carbohydrazide (3)}

General procedure: Equimolecular mixture of $1(3.21 \mathrm{~g}, 0.01 \mathrm{~mol})$ and either benzaldehyde $(1.08 \mathrm{~h}, 0.01 \mathrm{~mol}), p$-chlorobenzaldehyde $(1.38 \mathrm{~g}, 0.01 \mathrm{~mol})$, 4-methoxybenzaldehyde $(1.34 \mathrm{~g}, 0.01 \mathrm{~mol})$ or salicylaldehyde $(1.22 \mathrm{~g}, 0.01 \mathrm{~mol})$ in 1,4-dioxane $(20 \mathrm{~mL})$ containing piperidine $(0.50 \mathrm{~mL})$ was heated under reflux for $3 \mathrm{~h}$. The reaction mixture was left to cool then poured onto ice/water containing few drops of hydrochloric acid and the formed solid product, formed in each case, was collected by filtration.

2a. Colorless crystals of $\mathrm{mp} 233-225^{\circ} \mathrm{C}$ (ethanol). MS $(\mathrm{m} / \mathrm{z}, \%): 279\left(\mathrm{M}^{+}\right)$. IR $(\mathrm{KBr}), \mathrm{vcm}{ }^{-1}$ : 3452, $3336(\mathrm{NH}), 3059$ (aromatic $\mathrm{CH}), 2983\left(\mathrm{CH}_{3}\right), 2220(\mathrm{CN}), 1687(\mathrm{CO}), 1643(\mathrm{C}=\mathrm{N})$, $1636(\mathrm{C}=\mathrm{C}) .{ }^{1} \mathrm{H}$ NMR (300 MHz, DMSO-d, TMS): $1.06\left(\mathrm{~s}, 3 \mathrm{H}, \mathrm{CH}_{3}\right), 5.99(\mathrm{~s}, 1 \mathrm{H}, \mathrm{CH})$, 6.40-7.47 (m, 8H, C $\mathrm{H}_{5}$, furan $\left.\mathrm{CH}\right), 7.89(\mathrm{~s}, 1 \mathrm{H}, \mathrm{NH}) .{ }^{13} \mathrm{C}$ NMR $\left(300 \mathrm{MHz}\right.$, DMSO-d $d_{6}$, TMS): $\delta 18.7\left(\mathrm{CH}_{3}\right), 110.3,115.6,119.6,125.6,126.3,128.8,136.8,143.9$ (furan C, Benzene C), 111.0, 148.6 $(\mathrm{CH}=\mathrm{C}), 156.6(\mathrm{C}=\mathrm{N}), 168.3(\mathrm{C}=\mathrm{O})$. Anal. Calcd. for $\mathrm{C}_{16} \mathrm{H}_{13} \mathrm{~N}_{3} \mathrm{O}_{2}$ : C, 68.81; $\mathrm{H}, 4.69 ; \mathrm{N}, 15.05$. Found $\mathrm{C}, 68.77 ; \mathrm{H}, 4.49 ; \mathrm{N}, 14.72$.

2b. Colorless crystals of $\mathrm{mp} 199-201^{\circ} \mathrm{C}$ (ethanol). MS $(\mathrm{m} / \mathrm{z}, \%): 313\left(\mathrm{M}^{+}\right)$. IR $(\mathrm{KBr}), \mathrm{vcm}^{-1}$ : 3448, $3342(\mathrm{NH}), 3061$ (aromatic $\mathrm{CH}), 2980,2883\left(\mathrm{CH}_{3}\right), 2222(\mathrm{CN}), 1690$ (CO), 1646 $(\mathrm{C}=\mathrm{N}), 1632(\mathrm{C}=\mathrm{C}) .{ }^{1} \mathrm{H}$ NMR $\left(300 \mathrm{MHz}, \mathrm{DMSO}-d_{6}, \mathrm{TMS}\right): 1.02\left(\mathrm{~s}, 3 \mathrm{H}, \mathrm{CH}_{3}\right), 6.01(\mathrm{~s}, 1 \mathrm{H}$, $\mathrm{CH})$, 6.36-7.44 (m, 7H, C $\mathrm{H}_{4}$, furan $\left.\mathrm{CH}\right), 7.87(\mathrm{~s}, 1 \mathrm{H}, \mathrm{NH}) .{ }^{13} \mathrm{C}$ NMR $(300 \mathrm{MHz}$, DMSO-d TMS): $18.7\left(\mathrm{CH}_{3}\right), 112.4,114.2,118.9,125.6,126.3,127.9,136.8,144.6$ (furan $\mathrm{C}$, Benzene C), 111.0, 148.6 ( $\mathrm{CH}=\mathrm{C}), 153.6(\mathrm{C}=\mathrm{N}), 168.9(\mathrm{C}=\mathrm{O})$. Anal. Calcd. for $\mathrm{C}_{16} \mathrm{H}_{12} \mathrm{CIN}_{3} \mathrm{O}_{2}$ : C, 61.25; $\mathrm{H}, 3.86 ; \mathrm{N}, 13.39$. Found: $\mathrm{C}, 61.11 ; \mathrm{H}, 4.09 ; \mathrm{N}, 13.49$.

2c. Colorless crystals of $\mathrm{mp}>300^{\circ} \mathrm{C}$ (ethanol). $\mathrm{MS}(\mathrm{m} / \mathrm{z}, \%): 309\left(\mathrm{M}^{+}\right)$. IR $(\mathrm{KBr}), \mathrm{vcm}^{-1}$ : 3446, $3340(\mathrm{NH}), 3058$ (aromatic $\mathrm{CH}), 2977\left(\mathrm{CH}_{3}\right), 2220(\mathrm{CN}), 1668(\mathrm{CO}), 1644(\mathrm{C}=\mathrm{N})$, $1636(\mathrm{C}=\mathrm{C}) .{ }^{1} \mathrm{H}$ NMR (300 MHz, DMSO-d, $\left.\mathrm{TMS}\right): 1.03\left(\mathrm{~s}, 3 \mathrm{H}, \mathrm{CH}_{3}\right), 2.69\left(\mathrm{~s}, 3 \mathrm{H}, \mathrm{CH}_{3}\right)$, $6.04(\mathrm{~s}, 1 \mathrm{H}, \mathrm{CH}), 6.38-7.40\left(\mathrm{~m}, 7 \mathrm{H}, \mathrm{C}_{6} \mathrm{H}_{4}\right.$, furan $\left.\mathrm{CH}\right), 7.89(\mathrm{~s}, 1 \mathrm{H}, \mathrm{NH}) .{ }^{13} \mathrm{C} \mathrm{NMR}(300$ $\mathrm{MHz}$, DMSO-d $d_{6}$, TMS): 18.8, $24.3\left(2 \mathrm{CH}_{3}\right), 110.1,115.8,118.8,125.6,125.8,128.8,135.8$, 143.7(furan C, Benzene C), 110.9, $148.3(\mathrm{CH}=\mathrm{C}), 156.5(\mathrm{C}=\mathrm{N}), 168.0(\mathrm{C}=\mathrm{O})$. Anal. Calcd. for $\mathrm{C}_{17} \mathrm{H}_{15} \mathrm{~N}_{3} \mathrm{O}_{3}: \mathrm{C}, 66.01 ; \mathrm{H}, 4.89 ; \mathrm{N}, 13.58$. Found: $\mathrm{C}, 65.99 ; \mathrm{H}, 4.66 ; \mathrm{N}, 13.43$.

3. Colorless crystals of $\mathrm{mp} 229-231^{\circ} \mathrm{C}$ (ethanol). MS $(\mathrm{m} / \mathrm{z}, \%): 296\left(\mathrm{M}^{+}\right) . \mathrm{IR}(\mathrm{KBr}), \mathrm{vcm}^{-1}$ : 3452, $3330(\mathrm{NH}), 3059$ (aromatic $\mathrm{CH}), 2978\left(\mathrm{CH}_{3}\right), 1688-1685(2 \mathrm{CO}), 1640(\mathrm{C}=\mathrm{N}), 1638$ $(\mathrm{C}=\mathrm{C}) .{ }^{1} \mathrm{H}$ NMR $\left(300 \mathrm{MHz}, \mathrm{DMSO}-\mathrm{d}_{6}, \mathrm{TMS}\right): 1.21\left(\mathrm{~s}, 3 \mathrm{H}, \mathrm{CH}_{3}\right), 6.89(\mathrm{~s}, 1 \mathrm{H}$, coumarin $\mathrm{H}-4)$, 6.92-7.38 (m, 7H, C $\mathrm{H}_{4}$, furan $\left.\mathrm{CH}\right), 8.22(\mathrm{~s}, 1 \mathrm{H}, \mathrm{NH}) .{ }^{13} \mathrm{C} N M R\left(300 \mathrm{MHz}\right.$, DMSO-d $d_{6}$, TMS): $18.8\left(\mathrm{CH}_{3}\right), 110.3,119.6,120.8,121.3,121.8,122.6,124.7,128.3,136.8,143.9$ (furan $\mathrm{C}$, coumarin $\mathrm{C})$ ), $158.3(\mathrm{C}=\mathrm{N}), 166.5,168.8(2 \mathrm{C}=\mathrm{O})$. Anal. Calcd. for $\mathrm{C}_{16} \mathrm{H}_{12} \mathrm{~N}_{2} \mathrm{O}_{4}: \mathrm{C}$, 64.86; H, 4.08; N, 9.40. Found: C, 64.85; H, 4.33; N, 9.27.

\section{2-Cyano-N '-(1-furan-2-ylethylidene)-2-(phenylhydrazono)acetohydrazide (4a), 2-cyano-N'-(1-furan-2-ylethylidene)-2-[(4-methylphenyl)hydrazono]acetohydrazide (4b) and} 2-cyano-N'-(1-furan-2-ylethylidene)-2-[(4-methoxyphenyl)hydrazono]acetohydrazide (4c)

To a solution of compound $1(3.21 \mathrm{~g}, 0.01 \mathrm{~mol})$ in ethanol $(40 \mathrm{~mL})$ containing sodium acetate $(10.0 \mathrm{~g})$ either benzenediazonium chloride $(1.40 \mathrm{~g}, 0.01 \mathrm{~mol})$, 4-chlorobenzene- 
diazonium chloride $(1.75 \mathrm{~g}, 0.01 \mathrm{~mol})$ or 4-methylbenzenediazonium chloride $(1.55 \mathrm{~g}, 0.01$ $\mathrm{mol}$ ) with continuous stirring at $0{ }^{\circ} \mathrm{C}$. The solid product, formed in each case, was collected by filtration.

4a. Colorless crystals of $\mathrm{mp} 198-201^{\circ} \mathrm{C}$ (ethanol). MS $(\mathrm{m} / \mathrm{z}, \%): 295\left(\mathrm{M}^{+}\right)$. IR $(\mathrm{KBr}), \mathrm{vcm}^{-1}$ : 3466, $3328(2 \mathrm{NH}), 3062$ (aromatic CH), 2982, 2893( $\left.\mathrm{CH}_{3}\right), 2222(\mathrm{CN}) 1690(\mathrm{CO}), 1648$ $(\mathrm{C}=\mathrm{N}), 1635(\mathrm{C}=\mathrm{C}) .{ }^{1} \mathrm{H}$ NMR $\left(300 \mathrm{MHz}\right.$, DMSO- $\left.d_{6}, \mathrm{TMS}\right): 1.19\left(\mathrm{~s}, 3 \mathrm{H}, \mathrm{CH}_{3}\right), 6.87-7.39(\mathrm{~m}$, $8 \mathrm{H}, \mathrm{C}_{6} \mathrm{H}_{5}$, furan $\left.\mathrm{CH}\right), 7.99,8.20(2 \mathrm{~s}, 2 \mathrm{H}, 2 \mathrm{NH}) .{ }^{13} \mathrm{C}$ NMR (300 MHz, DMSO-d $\left.d_{6}, \mathrm{TMS}\right): 18.6$ $\left(\mathrm{CH}_{3}\right), 116.7(\mathrm{CN}), 110.3,119.6,120.4,120.8,121.3,123.0,128.0,134.6,144.2$ (furan $\mathrm{C}$, benzene $\mathrm{C}), 158.6(\mathrm{C}=\mathrm{N}), 165.8,168.9(2 \mathrm{C}=\mathrm{O})$. Anal. Calcd. for $\mathrm{C}_{15} \mathrm{H}_{13} \mathrm{~N}_{5} \mathrm{O}_{2}: \mathrm{C}, 61.01 ; \mathrm{H}$, 4.44; N, 23.72. Found: C, 60.88; H, 4.09; N, 23.3.

4b. Colorless crystals of $\mathrm{mp}>300{ }^{\circ} \mathrm{C}$ (ethanol). $\mathrm{MS}(\mathrm{m} / \mathrm{z}, \%): 329\left(\mathrm{M}^{+}\right)$. $\mathrm{IR}(\mathrm{KBr}), \mathrm{vcm}^{-1}$ : 3451, $3322(2 \mathrm{NH}), 3060$ (aromatic $\mathrm{CH}), 2980\left(\mathrm{CH}_{3}\right), 2221(\mathrm{CN}) 1690(\mathrm{CO}), 1646(\mathrm{C}=\mathrm{N})$, 1632 (C=C). ${ }^{1} \mathrm{H}$ NMR $\left(300 \mathrm{MHz}, \mathrm{DMSO}-\mathrm{d}_{6}, \mathrm{TMS}\right): 1.16\left(\mathrm{~s}, 3 \mathrm{H}, \mathrm{CH}_{3}\right), 7.03-7.37(\mathrm{~m}, 7 \mathrm{H}$, $\mathrm{C}_{6} \mathrm{H}_{4}$, furan $\mathrm{CH}$ ), 7.97, $8.22(2 \mathrm{~s}, 2 \mathrm{H}, 2 \mathrm{NH}) .{ }^{13} \mathrm{C}$ NMR (300 MHz, DMSO-d 6 , TMS): 18.7 $\left(\mathrm{CH}_{3}\right), 116.9(\mathrm{CN}), 111.8,119.2,120.6,121.8,123.6,128.5,133.9,140.5,144.2$ (furan $\mathrm{C}$, benzene $\mathrm{C})$, $158.6(\mathrm{C}=\mathrm{N}), 165.8,168.9(2 \mathrm{C}=\mathrm{O})$. Anal. Calcd. for $\mathrm{C}_{15} \mathrm{H}_{12} \mathrm{ClN}_{5} \mathrm{O}_{2}$ : $\mathrm{C}, 54.42$; $\mathrm{H}, 3.66 ; \mathrm{N}, 21.24$. Found: C, 54.64; H, 3.67; N, 21.24.

4c. Colorless crystals of $\mathrm{mp} 215-217^{\circ} \mathrm{C}$ (ethanol). $\mathrm{MS}(\mathrm{m} / \mathrm{z}, \%): 309\left(\mathrm{M}^{+}\right)$. IR $(\mathrm{KBr}), \mathrm{vcm}^{-1}$ : 3463, $3347(2 \mathrm{NH}), 3058$ (aromatic $\mathrm{CH}), 2982\left(\mathrm{CH}_{3}\right), 2222(\mathrm{CN}), 1693(\mathrm{CO}), 1642(\mathrm{C}=\mathrm{N})$, 1635 (C=C). ${ }^{1} \mathrm{H}$ NMR $\left(300 \mathrm{MHz}, \mathrm{DMSO}-\mathrm{d}_{6}, \mathrm{TMS}\right): 1.18\left(\mathrm{~s}, 3 \mathrm{H}, \mathrm{CH}_{3}\right), 2.88\left(\mathrm{~s}, 3 \mathrm{H}, \mathrm{CH}_{3}\right)$, $7.19-7.39\left(\mathrm{~m}, 7 \mathrm{H}, \mathrm{C}_{6} \mathrm{H}_{4}\right.$, furan $\left.\mathrm{CH}\right), 7.83,8.25(2 \mathrm{~s}, 2 \mathrm{H}, 2 \mathrm{NH}) .{ }^{13} \mathrm{C}$ NMR $(300 \mathrm{MHz}$, DMSO$d_{6}$, TMS): $18.8,120.7\left(2 \mathrm{CH}_{3}\right), 116.7(\mathrm{CN}), 110.6,119.4,120.6,121.0,121.3,123.2$, 128.2,134.8, 144.0 (furan C, benzene C), $158.9(\mathrm{C}=\mathrm{N}), 166.2,168.7(2 \mathrm{C}=\mathrm{O})$. Anal. Calcd. for $\mathrm{C}_{16} \mathrm{H}_{15} \mathrm{~N}_{5} \mathrm{O}_{2}: \mathrm{C}, 62.13 ; \mathrm{H}, 4.89 ; \mathrm{N}, 22.64$. Found: $\mathrm{C}, 62.09 ; \mathrm{H}, 4.89 ; \mathrm{N}, 22.74$.

\section{6-Amino-1-[(1-furan-2-ylethylidene)amino]-2-oxo-4-phenyl-1,2-dihydropyridine- 3,5-dicarbonitrile (6a) and ethyl 2-amino-5-cyano-1-[(1-furan-2-ylethylidene)amino]-6-oxo-4-phenyl- 1,6-dihydropyridine-3-carboxylate (6b)}

Method (A): To a solution of compound $1(3.21 \mathrm{~g}, 0.01 \mathrm{~mol})$ in 1,4-dioxane $(40 \mathrm{~mL})$ containing triethylamine $(1.0 \mathrm{~mL})$, either benzalmalononitrile $(1.54 \mathrm{~g}, 0.01 \mathrm{~mol})$ or ethyl benzalacetate $(2.01 \mathrm{~g}, 0.01 \mathrm{~mol})$ was added. The reaction mixture, in each case was heated under reflux for $3 \mathrm{~h}$ then poured onto ice/water and the formed solid product was collected by filtration.

Method (B): To a solution of compound $2 \mathrm{a}(3.21 \mathrm{~g}, 0.01 \mathrm{~mol})$ in 1,4-dioxane $(40 \mathrm{~mL})$ containing triethylamine $(1.0 \mathrm{~mL})$ either malononitrile $(0.66 \mathrm{~g}, 0.01 \mathrm{~mol})$ or ethyl cyanoacetate $(1.13 \mathrm{~g}, 0.01 \mathrm{~mol})$ was added. The reaction mixture was heated under reflux for $6 \mathrm{~h}$ the poured onto ice/water and the formed solid product was collected by filtration.

6a. Colorless crystals of $\mathrm{mp} 188-190^{\circ} \mathrm{C}\left(1,4\right.$ dioxane). $\mathrm{MS}(\mathrm{m} / \mathrm{z}, \%): 343\left(\mathrm{M}^{+}\right)$. IR $(\mathrm{KBr})$, $\mathrm{vcm}^{-1}: 3460,3387\left(\mathrm{NH}_{2}\right), 3054$ (aromatic $\left.\mathrm{CH}\right), 2989\left(\mathrm{CH}_{3}\right), 2225,2221(2 \mathrm{CN}), 1690(\mathrm{CO})$, $1640(\mathrm{C}=\mathrm{N}), 1637(\mathrm{C}=\mathrm{C}) .{ }^{1} \mathrm{H}$ NMR $\left(300 \mathrm{MHz}, \mathrm{DMSO}^{-} \mathrm{d}_{6}, \mathrm{TMS}\right): 1.05\left(\mathrm{~s}, 3 \mathrm{H}, \mathrm{CH}_{3}\right), 4.87(\mathrm{~s}$, $\left.2 \mathrm{H}, \mathrm{NH}_{2}\right), 7.28-7.41\left(\mathrm{~m}, 8 \mathrm{H}, \mathrm{C}_{6} \mathrm{H}_{5}\right.$, furan $\left.\mathrm{CH}\right) .{ }^{13} \mathrm{C}$ NMR (300 MHz, DMSO-d 6 , TMS): 18.8 $\left(\mathrm{CH}_{3}\right), 116.9,117.3(2 \mathrm{CN}), 111.6,119.3,120.4,121.4,122.7,123.0,126.2,134.7,145.6$ 
(furan $\mathrm{C}$, benzene $\mathrm{C}), 158.7(\mathrm{C}=\mathrm{N}), 165.2(\mathrm{C}=\mathrm{O})$. Anal. Calcd. for $\mathrm{C}_{19} \mathrm{H}_{13} \mathrm{~N}_{5} \mathrm{O}_{2}$ : $\mathrm{C}, 66.47 ; \mathrm{H}$, 3.82; N, 20.40. Found:. C, 66.61; H, 3.49; N, 20.69.

6b. Colorless crystals of $\mathrm{mp}>300^{\circ} \mathrm{C}$ (ethanol). MS (m/z, \%): $390\left(\mathrm{M}^{+}\right)$. IR $(\mathrm{KBr}), \mathrm{vcm}^{-1}$ : 3452, $3376\left(\mathrm{NH}_{2}\right), 3056$ (aromatic $\left.\mathrm{CH}\right), 2985,2888\left(\mathrm{CH}_{3}, \mathrm{CH}_{2}\right), 2227,(\mathrm{CN}), 1689,1686$ (2CO), $1638(\mathrm{C}=\mathrm{N}), 1632(\mathrm{C}=\mathrm{C}) .{ }^{1} \mathrm{H}$ NMR (300 MHz, DMSO-d, $\left.\mathrm{TMS}\right): 1.09$ (s, 3H, $\left.\mathrm{CH}_{3}\right)$, $1.16\left(\mathrm{t}, 3 \mathrm{H}, \mathrm{J}=6.79 \mathrm{~Hz}, \mathrm{CH}_{3}\right), 4.24\left(\mathrm{q}, 2 \mathrm{H}, \mathrm{J}=6.79 \mathrm{~Hz}, \mathrm{CH}_{2}\right), 4.79\left(\mathrm{~s}, 2 \mathrm{H}, \mathrm{NH}_{2}\right), 7.30-7.45$ (m, 8H, $\mathrm{C}_{6} \mathrm{H}_{5}$, furan $\left.\mathrm{CH}\right) .{ }^{13} \mathrm{C}$ NMR (300 MHz, DMSO-d, TMS): 18.8, $20.8\left(2 \mathrm{CH}_{3}\right), 62.6$ $\left(\mathrm{CH}_{2}\right), 116.6(\mathrm{CN}), 110.9,119.0,120.8,121.6,122.9,123.7,126.0,134.9,144.3$ (furan $\mathrm{C}$, benzene C), $158.8(\mathrm{C}=\mathrm{N}), 165.0(\mathrm{C}=\mathrm{O})$. Anal. Calcd. for $\mathrm{C}_{21} \mathrm{H}_{18} \mathrm{~N}_{4} \mathrm{O}_{4}: \mathrm{C}, 64.08 ; \mathrm{H}, 4.62 ; \mathrm{N}$, 14.16. Found: $\mathrm{C}, 64.61 ; \mathrm{H}, 4.65 ; \mathrm{N}, 14.35$.

\section{4,6-Diamino-1-[(1-furan-2-ylethylidene)amino]-2-oxo-1,2-dihydropyridine- 3-carbonitrile (7a) and ethyl 4-amino-5-cyano-1-[(1-furan-2-ylethylidene)amino]-6-oxo-1,6-dihydropyridine- 2-carboxylate (7b)}

Equimolecular amounts of compound $1(0.01 \mathrm{~mol})$ and either malononitrile $(0.66 \mathrm{~g}, 0.01$ mol) or ethyl cyanoacetate $(1.13 \mathrm{~g}, 0.01 \mathrm{~mol})$ in 1,4-dioxane $(30 \mathrm{~mL})$ containing triethylamine $(1.5 \mathrm{~mL})$ was heated under reflux for $5 \mathrm{~h}$. The reaction mixture was left to cool and evaporated under vacuum. The remaining product was triturated with ethanol and the formed solid product was collected by filtration.

7a. Colorless crystals of $\mathrm{mp} 202-204^{\circ} \mathrm{C}$ (ethanol). MS $(\mathrm{m} / \mathrm{z}, \%): 257\left(\mathrm{M}^{+}\right) . \mathrm{IR}(\mathrm{KBr}), \mathrm{vcm}^{-1}$ : 3473, $3351\left(2 \mathrm{NH}_{2}\right), 3058$ (aromatic $\left.\mathrm{CH}\right), 2987\left(\mathrm{CH}_{3}\right), 2223,(\mathrm{CN}), 1687(\mathrm{CO}), 1641(\mathrm{C}=\mathrm{N})$, $1634(\mathrm{C}=\mathrm{C}) .{ }^{1} \mathrm{H}$ NMR (300 MHz, DMSO-d, $\left.\mathrm{TMS}\right): 1.11\left(\mathrm{~s}, 3 \mathrm{H}, \mathrm{CH}_{3}\right), 4.81,4.93(2 \mathrm{~s}, 4 \mathrm{H}$, $\left.2 \mathrm{NH}_{2}\right), 6.61(\mathrm{~s}, 1 \mathrm{H}$, pyridine $\mathrm{H}-3), 6.82-6.91(\mathrm{~m}, 3 \mathrm{H}$, furan $\mathrm{CH}) .{ }^{13} \mathrm{C} N M R(300 \mathrm{MHz}$, DMSO- $d_{6}$, TMS): $18.6\left(\mathrm{CH}_{3}\right), 116.9(\mathrm{CN}), 112.6,118.8,120.3,121.9,122.6,130.2,134.8$, 140.6 (furan $\mathrm{C}$, pyridine $\mathrm{C}$ ), $159.4(\mathrm{C}=\mathrm{N}), 165.8(\mathrm{C}=\mathrm{O})$. Anal. Calcd. for $\mathrm{C}_{12} \mathrm{H}_{11} \mathrm{~N}_{5} \mathrm{O}_{2}$ : C,56.03; H, 4.31; N, 27.22. Found: C, 55.84; H, 4.33; N, 26.81.

7b. Colorless crystals of $\mathrm{mp} 176-177^{\circ} \mathrm{C}$ (ethanol). MS $(\mathrm{m} / \mathrm{z}, \%): 258\left(\mathrm{M}^{+}\right)$. IR $(\mathrm{KBr}), \mathrm{vcm}^{-1}$ : 3530, $3380\left(\mathrm{OH}, \mathrm{NH}_{2}\right), 3053$ (aromatic $\left.\mathrm{CH}\right), 2989\left(\mathrm{CH}_{3}\right)$ 2221, (CN), 1686 (CO), 1640 $(\mathrm{C}=\mathrm{N}), 1631(\mathrm{C}=\mathrm{C}) .{ }^{1} \mathrm{H}$ NMR (300 MHz, DMSO-d, $\left.\mathrm{TMS}\right): 1.13\left(\mathrm{~s}, 3 \mathrm{H}, \mathrm{CH}_{3}\right), 4.83(\mathrm{~s}, 2 \mathrm{H}$, $\left.\mathrm{NH}_{2}\right), 6.48(\mathrm{~s}, 1 \mathrm{H}$, pyridine $\mathrm{H}-3), 6.80-6.93(\mathrm{~m}, 4 \mathrm{H}$, furan $\mathrm{CH}), 10.25(\mathrm{~s}, 1 \mathrm{H}, \mathrm{OH}) .{ }^{13} \mathrm{C} N M R$ (300 MHz, DMSO-d 6 , TMS): $18.2\left(\mathrm{CH}_{3}\right), 116.5(\mathrm{CN}), 112.9,119.1,120.6,122.3,122.9$, 130.7, 140.3, 144.8 (furan $C$, pyridine $C$ ), $159.6(C=N), 165.3(C=O)$. Anal. Calcd. for $\mathrm{C}_{12} \mathrm{H}_{10} \mathrm{~N}_{4} \mathrm{O}_{3}$ : C, 55.81; $\mathrm{H}, 3.90 ; \mathrm{N}, 21.70$. Found: $\mathrm{C}, 55.63 ; \mathrm{H}, 4.07 ; \mathrm{N}, 21.82$.

\section{3,5-Diamino-4-cyano-N'-(1-furan-2-ylethylidene)thiophene-2-carbohydrazide (8a), ethyl 2,4-diamino-5-\{[2-(1-furan-2-ylethylidene)hydrazino]carbonyl\}thiophene- 3-carboxylate (8b) and 3-amino-N '-(1-furan-2-ylethylidene)-4,5,6,7-tetrahydro-1-benzothiophene- 2-carbohydrazide (9)}

General procedure: To a solution of compound $1(3.21 \mathrm{~g}, 0.01 \mathrm{~mol})$ in absolute ethanol (50 $\mathrm{mL})$ containing triethylamine $(1.0 \mathrm{~mL})$ either malononitrile $(0.66 \mathrm{~g}, 0.01 \mathrm{~mol})$, ethyl cyanoacetate $(1.13 \mathrm{~g}, 0.01 \mathrm{~mol})$ or cyclohexanone $(0.98 \mathrm{~g}, 0.01 \mathrm{~mol})$ together with elemental sulfur $(0.32 \mathrm{~g}, 0.01 \mathrm{~mol})$ were added. The whole reaction mixture was heated 
under reflux for $1 \mathrm{~h}$ then poured onto ice/water mixture and the formed solid product, in each case, was collected by filtration.

8a. Colorless crystals of $\mathrm{mp}>300{ }^{\circ} \mathrm{C}$ (ethanol). MS $(\mathrm{m} / \mathrm{z}, \%): 289\left(\mathrm{M}^{+}\right)$. IR $(\mathrm{KBr}), \mathrm{vcm}^{-1}$ : 3462, $3326\left(2 \mathrm{NH}_{2}, \mathrm{NH}\right), 3056$ (aromatic $\left.\mathrm{CH}\right), 2978\left(\mathrm{CH}_{3}\right) 2226(\mathrm{CN}), 1683$ (CO), 1644 $(\mathrm{C}=\mathrm{N}), 1635(\mathrm{C}=\mathrm{C}) .{ }^{1} \mathrm{H}$ NMR (300 MHz, DMSO-d, $\left.\mathrm{TMS}\right): 1.08$ (s, 3H, $\left.\mathrm{CH}_{3}\right), 4.80,4.93$ (2s, $\left.4 \mathrm{H}_{2} \mathrm{NH}_{2}\right), 6.78-6.90(\mathrm{~m}, 4 \mathrm{H}$, furan $\mathrm{CH}), 8.1(\mathrm{~s}, 1 \mathrm{H}, \mathrm{NH}) .{ }^{13} \mathrm{C} \mathrm{NMR}(300 \mathrm{MHz}$, DMSO-d TMS): $18.7\left(\mathrm{CH}_{3}\right), 115.9(\mathrm{CN}), 110.2,112.5,116.9,120.5,124.4,132.7,134.9,140.4$ (furan, thiophene C), $156.9(\mathrm{C}=\mathrm{N}), 165.6(\mathrm{C}=\mathrm{O})$. Anal. Calcd. for $\mathrm{C}_{12} \mathrm{H}_{11} \mathrm{~N}_{5} \mathrm{O}_{2} \mathrm{~S}: \mathrm{C}, 49.82$; H, 3.83; N, 24.21;S, 11.08. Found: C, 49.67; H, 3.59; N,24.07; S, 10.83.

8b. Colorless crystals of $\mathrm{mp} 209-211^{\circ} \mathrm{C}$ (ethanol). MS $(\mathrm{m} / \mathrm{z}, \%): 336\left(\mathrm{M}^{+}\right)$. IR $(\mathrm{KBr}), \mathrm{vcm}{ }^{-}$ 1:3462, $3326\left(2 \mathrm{NH}_{2}, \mathrm{NH}\right), 3056$ (aromatic $\left.\mathrm{CH}\right), 2978\left(\mathrm{CH}_{3}\right) 2880\left(\mathrm{CH}_{2}\right), 2226(\mathrm{CN}), 1683$ (CO), $1644(\mathrm{C}=\mathrm{N}), 1635(\mathrm{C}=\mathrm{C}) .{ }^{1} \mathrm{H}$ NMR (300 MHz, DMSO-d, TMS): $1.07\left(\mathrm{~s}, 3 \mathrm{H}, \mathrm{CH}_{3}\right)$, $1.14\left(\mathrm{t}, 3 \mathrm{H}, \mathrm{J}=7.36 \mathrm{~Hz}, \mathrm{CH}_{3}\right), 4.22\left(\mathrm{q}, 2 \mathrm{H}, \mathrm{J}=7.36 \mathrm{~Hz}, \mathrm{CH}_{2}\right), 4.79,4.87\left(2 \mathrm{~s}, 4 \mathrm{H}, 2 \mathrm{NH}_{2}\right)$, 6.63-6.85 (m, 3H, furan $\mathrm{CH}), 8.22(\mathrm{~s}, 1 \mathrm{H}, \mathrm{NH}) .{ }^{13} \mathrm{C} \mathrm{NMR}\left(300 \mathrm{MHz}, \mathrm{DMSO}-\mathrm{d}_{6}, \mathrm{TMS}\right): 18.3$, $24.8\left(2 \mathrm{CH}_{3}\right), 62.6\left(\mathrm{CH}_{2}\right), 110.8,112.2,116.6,120.4,124.4,132.9,134.7,140.4$ (furan, thiophene C), $156.6(\mathrm{C}=\mathrm{N}), 165.6,166.0(2 \mathrm{C}=\mathrm{O})$. Anal. Calcd. for $\mathrm{C}_{14} \mathrm{H}_{16} \mathrm{~N}_{4} \mathrm{O}_{4} \mathrm{~S}: \mathrm{C}, 49.99$; H, 4.79; N, 16.66;S, 9.53. Found: C, 49.85; H, 4.33; N,1627; S, 9.71.

9. Colorless crystals of $\mathrm{mp} 190-191^{\circ} \mathrm{C}$ (ethanol). MS (m/z, \%): $303\left(\mathrm{M}^{+}\right)$. IR $(\mathrm{KBr}), \mathrm{vcm}^{-1}$ : 3459, $3421\left(\mathrm{NH}_{2}, \mathrm{NH}\right), 3049$ (CH aromatic), 2988, $2876\left(\mathrm{CH}_{3}, \mathrm{CH}_{2}\right), 1686$ (CO), 1644 $(\mathrm{C}=\mathrm{N}), 1632(\mathrm{C}=\mathrm{C}) .{ }^{1} \mathrm{H}$ NMR (300 MHz, DMSO-d, $\left.\mathrm{TMS}\right): 1.11\left(\mathrm{CH}_{3}\right), 2.21-2.26(\mathrm{~m}, 4 \mathrm{H}$, $2 \mathrm{CH} 2), 2.30-2.36\left(\mathrm{~m}, 4 \mathrm{H}, 2 \mathrm{CH}_{2}\right), 4.78\left(\mathrm{~s}, 2 \mathrm{H}, \mathrm{NH}_{2}\right), 6.48-6.82(\mathrm{~m}, 3 \mathrm{H}$, furan $\mathrm{CH}), 8.01(\mathrm{~s}$, $1 \mathrm{H}, \mathrm{NH}) .{ }^{13} \mathrm{C} \operatorname{NMR}\left(300 \mathrm{MHz}, \mathrm{DMSO}-d_{6}, \mathrm{TMS}\right): 18.6(\mathrm{CH} 3), 20.3,123.5,124.2(4 \mathrm{CH} 2)$, 120.5, 122.3, 124.6, 126.8, 134.2, 140.6 (thiophene, furan $\mathrm{C}), 156.3(\mathrm{C}=\mathrm{N}), 165.9(\mathrm{C}=\mathrm{O})$. Anal. Calcd. for $\mathrm{C}_{15} \mathrm{H}_{17} \mathrm{~N}_{3} \mathrm{O}_{2} \mathrm{~S}$ : C, 59.38; $\mathrm{H}, 5.65 ; \mathrm{N}, 13.85 ; \mathrm{S}, 10.57$. Found: $\mathrm{C}, 59.26 ; \mathrm{H}$, 5.52; N,13.55; S, 10.33 .

\section{4-amino-N '-(1-furan-2-ylethylidene)-3-phenyl-2-thioxo-2,3-dihydro-1,3-thiazole- 5-carbohydrazide (10)}

To a solution of compound $1(3.21 \mathrm{~g}, 0.01 \mathrm{~mol})$ in absolute ethanol (30 $\mathrm{mL})$ containing triethylamine $(1.0 \mathrm{~mL})$ and elemental sulfur $(0.32 \mathrm{~g}, 0.01 \mathrm{~mol})$, phenylisothiocyanate $(1.30$ $\mathrm{g}, 0.01 \mathrm{~mol}$ ) was added. The reaction mixture was heated under reflux for $3 \mathrm{~h}$ then left to cool. The solid product formed upon pouring onto ice/water was collected by filtration.

10. Colorless crystals of $\mathrm{mp} 160{ }^{\circ} \mathrm{C}$ (acetic acid). MS $(\mathrm{m} / \mathrm{z}, \%): 358\left(\mathrm{M}^{+}\right)$. IR $(\mathrm{KBr}), \mathrm{vcm}^{-1}$ : 3466, $3329\left(\mathrm{NH}_{2}, \mathrm{NH}\right), 3020\left(\mathrm{CH}\right.$ aromatic), $2986\left(\mathrm{CH}_{3}\right), 1644(\mathrm{C}=\mathrm{N}), 1629(\mathrm{C}=\mathrm{C}), 1205-$ $1196(\mathrm{C}=\mathrm{S}) .{ }^{1} \mathrm{H}$ NMR (300 MHz, DMSO-d ${ }_{6}$, TMS): $1.13\left(\mathrm{CH}_{3}\right), 4.70\left(\mathrm{~s}, 2 \mathrm{H}, \mathrm{NH}_{2}\right), 6.49-6.77$ (m, 3H, furan $\mathrm{CH}), 7.29-7.35\left(\mathrm{~m}, 5 \mathrm{H}, \mathrm{C}_{6} \mathrm{H}_{5}\right), 8.11(\mathrm{~s}, 1 \mathrm{H}, \mathrm{NH}) .{ }^{13} \mathrm{C} \mathrm{NMR}(300 \mathrm{MHz}$, DMSO$d_{6}$, TMS): 18.5 (CH3), 73.9 (thiazole C-5), 115.6, 118.3, 120.9, 122.4, 126.8, 128.4, 130.5, 133.5, 140.1 (furan $\mathrm{C}$, thiazole $\mathrm{C}-6$, benzene $\mathrm{C}$ ), $156.7(\mathrm{C}=\mathrm{N}), 166.1(\mathrm{C}=\mathrm{O}), 188.0(\mathrm{C}=\mathrm{S})$. Anal. Calcd. for $\mathrm{C}_{16} \mathrm{H}_{14} \mathrm{~N}_{4} \mathrm{O}_{2} \mathrm{~S}_{2}$ : $\mathrm{C}, 53.61 ; \mathrm{H}, 3.94 ; \mathrm{N}, 15.63 ; \mathrm{S}, 17.89$. Found: $\mathrm{C}, 53.28 ; \mathrm{H}$, 3.55; N, 15.73; S, 17.57.

\section{Antimicrobial Activity}

The in vitro antimicrobial activity of the newly heterocyclic derivatives against two strains of Gram positive bacteria (Bacillus subtilis CECT 498 and Bacillus cereus CECT 148), one 
strain of Gram negative bacteria (Escherichia coli ECT 101 ) and Candida albicans CECT 1394 as a representative species of fungi was investigated. The newly synthesized products were dissolved in aqueous ethanol to give a logarithmic series of concentrations from 2 to $256 \mathrm{mg} / \mathrm{L}$ upon tenfold dilution with the growth medium and spore suspension of the test fungi. The toxicity of compounds was determined via a pipette additions into the wells of multi-well slides, followed with $25 \mu \mathrm{L}$ of the culture medium. The incubated slides were then incubated at $25{ }^{\circ} \mathrm{C}$ until short germ tubes appeared; approximately $50 \mu \mathrm{m}$ in length (at $0 \mathrm{~h}$ ) was measured. Five $\mu \mathrm{L}$ volumes of the prepared compound test solutions were added to the incubated wells, one control well on each slide being treated with solvent only. The slides were then returned to the incubator until germ tubes $400 \mu \mathrm{m}$ $( \pm 50 \mu \mathrm{m})$ long were visible in the control wells. Further growth was arrested by the addition of lactophenol aniline blue to each of the wells. The minimal inhibitory concentration (MIC in $\mu \mathrm{g} \mathrm{mL}^{-1}$ ) was determined using an adaptation of agar streak dilution method based on radial diffusion. Under the same conditions ampicillin (antibacterial) and cycloheximide (antifungal) were used as standards. The MIC was considered to be the lowest concentration of the tested compound (in dimethylformamide) which inhibits growth of bacteria or fungi on the plate (Table 1). The diameters of the inhibition zones corresponding to the MICs are also presented in Table 1.

Tab. 1. Antimicrobial activities of the tested compounds

\begin{tabular}{lllll}
\hline Comp. & \multicolumn{4}{c}{ MIC $\left(\boldsymbol{\mu g m L}^{-1}\right.$ ) (zone of inhibition, mm) } \\
No. & E. coli & B. cereus & B. subtilis & C. albicans \\
\cline { 2 - 5 } 1 & $12.50(6)$ & $22.52(8)$ & $20.55(4)$ & $8.65(4)$ \\
2a & - & $6.05(15)$ & $21.01(8)$ & $33.23(6)$ \\
2b & - & $11.32(3)$ & $18.22(8)$ & $12.40(4)$ \\
2c & - & $12.30(4)$ & $3.72(6)$ & $16.58(12)$ \\
3 & - & $22.38(8)$ & $22.83(6)$ & $24.18(3)$ \\
4a & - & $19.15(4)$ & $20.46(9)$ & $0.40(10)$ \\
4b & $16.45(6)$ & $18.23(5)$ & $22.45(8)$ & $40.18(6)$ \\
4c & - & $18.12(5)$ & $12.25(2)$ & $23.64(6)$ \\
6a & - & $10.05(6)$ & $16.42(2)$ & $4.55(10)$ \\
6b & - & $0.03(9)$ & $4.13(10)$ & $0.61(6)$ \\
7a & - & $18.24(7)$ & $6.18(4)$ & $0.40(5)$ \\
7b & - & $6.25(15)$ & $22.01(8)$ & $30.23(6)$ \\
8a & - & $16.22(3)$ & $18.32(8)$ & $14.40(4)$ \\
8b & - & $10.38(4)$ & $6.22(6)$ & $12.55(12)$ \\
9 & - & $25.36(8)$ & $23.63(6)$ & $26.12(3)$ \\
10 & - & $21.15(4)$ & $23.16(9)$ & $38.15(4)$ \\
Ampicillin & $6.25(10)$ & $3.13(15)$ & $12.50(10)$ & - \\
Cycloheximide & - & - & - & $12.50(15)$
\end{tabular}

\section{Authors' Statement}

\section{Competing Interests}

The authors declare no conflict of interest. 


\section{References}

[1] Karegoudar P, Parasa DJ, Ashok M, Mahalinga M, Poojary B, Holla BS.

Synthesis, antimicrobial and ant-inflammatory activities of some 1,2,4-triazolo[3,4-b][1,3,4]thiazoles

and 1,2,4-triazolo[3,4-b]thiadiazines bearing trichlorophenyl moiety.

Eur J Med Chem. 2008; 43: 208-815.

doi:10.1016/j.ejmech.2007.06.026

[2] Pomarnacka, E, Bednarski PJ, Reszka P, Borys E. D., Bienczak A, Werel W, Halasa R.

Synthesis and biological activity of new 2-amino-8-chloro-5,5-dioxo[1,2,4]triazolo[2,3-

b] [1,4,2]benzodithiazines.

Eur J Med Chem. 2006; 41: 633-639.

doi:10.1016/j.ejmech.2005.11.009

[3] Padmavathi V, Reddy GS, Padmaia A, Kondaiah P, Shazia A.

Synthesis, antimicrobial and cytotoxic activities of 1,3,4-oxadiazoles, 1,3,4-thiadiazoles and 1,2,4triazoles.

Eur J Med Chem. 2009; 44: 206-2112.

doi:10.1016/j.ejmech.2008.10.012

[4] Ragavendran JV, Sriram D, Patel SK, Reddy IV, Bharathwajan N, Stables J, Yogeeswari P.

Design and synthesis of anticonvulsants from a combined phthalimide-GARA-anilide and hydrazone pharmacophore.

Eur J Med Chem. 2007; 42: 146-151.

doi:10.1016/j.ejmech.2006.08.010

[5] Rahman VP, Mukhtar S, Ansari WH, Lemiere G.

Syhthesis,stereochemistry and biological activity of some novel long alkyl chain substituted thiazolidin4-ones and thiazan-4-one from 10-undecenoic acid hydrazide.

Eur J Med Chem. 2005; 40: 173-184.

doi:10.1016/j.ejmech.2004.10.003

[6] Zora M, Gormen M.

Synthesis of ferrocenyl pyrazoles by the reaction of (2-formyl-1-chlorovinyl)ferrocene.

J Organomet Chem. 2007; 692: 5026-5032.

doi:10.1016/j.jorganchem.2007.07.029

[7] Ito T, Fraser IP, Yeo Yoon, Highley CB, Bellas E, Kohane DS.

Anti-inflammatory function of an in situ cross-linkable conjugate hydrogel of hyaluronic acid and dexamethasone.

Biomaterials. 2007; 28: 1778-1786.

doi:10.1016/j.biomaterials.2006.12.012

[8] Acharya BN, Saraswat D, Kaushik MP.

Pharmacophore based discovery of potential antimalarial agent targeting haem detoxification pathway.

Eur J Med Chem. 2008; 43: 2840-2852.

doi:10.1016/j.ejmech.2008.02.005

[9] Xia Yong, Fan CD, Zhao BX, Zhao J, Shin DS, Miao JY.

Synthesis and structure relationships of novel 1-arylmethyl-3-aryl-1H-pyrazole-5-carbohydrazide

hydrazone derivatives as potential agents against A549 lung cancer cells.

Eur J Med Chem. 2008; 43: 2347-2353.

doi:10.1016/j.ejmech.2008.01.021

[10] Imramovsky A, Polanc S, Vinsova J, Kocevar M, Jampilek J, Reckova Z, Kaustova J.

New modification of anti-tubercular active molecules.

Bioorg Med Chem. 2007; 15: 2551-2559.

doi:10.1016/j.bmc.2007.01.051

[11] Dumciute J, Martynaitis V, Holzer W, Mangelinckx S, Kimpe ND, Sackus A.

Synthesis and ring transformations of 1-amino-1,2,3,9a-[1,2-a]indol-2(9H)-ones.

Tetrahedron. 2006; 62: 3309-3319.

doi:10.1016/j.tet.2006.01.054 
[12] Bedia KB, Elcin O, Deda U, Fatma K, Nathaly S, Sevim R, Dimoglo A.

Synthesis and characterization of novel hydrazide-hydrazones and the study of their structureantituberculosis.

Eur J Med Chem. 2006; 41: 1253-1261.

doi:10.1016/j.ejmech.2006.06.009

[13] Melnyk P, Leroux V, Sergheraert C, Grellier P.

Design, synthesis and in vitro antimalarial of an acylhydrazone library.

Bioorg Med Chem Lett. 2006; 16: 31-35.

doi:10.1016/j.bmcl.2005.09.058

[14] Richardson DR, Ponka P.

Pyridoxal isonicotinoyl hydrazone and its analogs: Potential orally effective iron-chelating agents for the treatment of iron overload disease.

J Lab. Clin Med. 1998; 131: 306-315.

doi:10.1016/S0022-2143(98)90180-9

[15] Kucukguzel G, Kocatepe A, Clercq ED, Sahin F, Gulluce M.

Synthesis and biological activity of 4-thiazolidinones, thiosemicarbazides derived from diflunisal hydrazide.

Eur J Med Chem. 2006; 41: 353-359.

doi:10.1016/j.ejmech.2005.11.005

[16] Zhou JF, Gong GX, Zhu FX, Zhi SJ.

Microwave promoted one-pot synthesis of 3-(2'-amino-3'-cyano-4'-arylpyrid-6'-yl) coumarins.

Chin Chem Lett. 2009; 20: 37-39.

doi:10.1016/j.cclet.2008.10.006

[17] Volmajer J, Toplak R, Leban I, Le Marechal AM.

Synthesis of new iminocoumarins and their transformations into $\mathrm{N}$-chloro and hydrazono compounds. Tetrahedron. 2005; 61: 7012-7021.

doi:10.1016/j.tet.2005.05.020

[18] Mckibben BP, Cartwright CH, Castelhano L.

Practical synthesis of tetrasubstituted thiophenes for use in compound libraries.

Tetrahedron Lett.1999; 40: 5471-5474.

doi:10.1016/S0040-4039(99)01108-9

[19] Scrowston RM.

Recent advances in the chemistry of benzo[b]thiophenes.

Adv Heterocycl Chem. 1981; 29: 171-249.

doi:10.1016/S0065-2725(08)60788-6

[20] Tormos GV, Khodorkovsky YU, Neilands OY, Belyakov SV.

A novel cyclocondensation of xanthates containing active methylene groups with isothiocyanates.Spectral data and X-ray structures of the products.

Tetrahedron. 1992; 48: 6863-6874.

doi:10.1016/S0040-4020(01)89877-X 